\title{
Casting quality model for casted aluminium silicon carbide based on non-linear thermal expansion
}

\begin{abstract}
This paper presents the design and development of a model for predicting the casting quality of aluminium silicon carbide based on the first-order shear deformation theory (FSDT) and thermal expansion behaviour. The coefficient of thermal expansion (CTEs) of casted aluminium silicon carbide fibre reinforced material was significantly influenced by the thermal stresses and interfaces between matrix and fibres. The thermal expansion behaviour of the casted aluminium silicon carbide fibre reinforced composite relies on the thermal expansion of the fibres, and influenced by the onset of interfacial strength and residual stress state. The validation shows a good agreement with surface roughness. In order to determine the performance of the model, the analysis of variance (ANOVA) was presented by using SPSS. The performance of casting quality model shows the correlation at the high level of accuracy $99.9 \%$ with confidence level of $95 \%$ between the experiment and the model.
\end{abstract}

Keyword: Product; Casting; Technology; Quality; Model; Materials; Aluminium; Silicon; Carbide; Non-linear; Thermal; Expansion; Production tooling; Surface roughness 